\title{
Suppression of hepatic tumor growth and metastasis by metronomic therapy in a rat model of hepatocellular carcinoma
}

\author{
Jeong Won Jang ${ }^{1,2}$, Seong Tae Park', \\ Jung Hyun Kwon ${ }^{1,2}$, Chan Ran You ${ }^{1,2}$, \\ Jong Young Choi ${ }^{1,2}$, Chan-Kwon Jung ${ }^{3}$, \\ Si Hyun Bae ${ }^{1,2,4}$ and Seung Kew Yoon ${ }^{1,2,4}$ \\ ${ }^{1}$ Department of Internal Medicine \\ WHO Collaborating for Reference \\ Research on Viral Hepatitis \\ ${ }^{2}$ Department of Internal Medicine \\ ${ }^{3}$ Department of Hospital Pathology \\ The Catholic University of Korea \\ Seoul, Korea \\ ${ }^{4}$ Corresponding author: Tel, 82-2-2258-6020; \\ Fax, 82-2-3481-4025; E-mail, yoonsk@ catholic.ac.kr (S.K.Y); \\ E-mail, baesh@ catholic.ac.kr (S.H.B) \\ DOI 10.3858/emm.2011.43.5.033
}

Accepted 22 March 2011

Available Online 8 April 2011

Abbreviations: CTX, cyclophosphamide; DEN, diethylnitrosamine; HCC, hepatocellular carcinoma; MET, metronomic; MMP, matrix metalloproteinase; MTD, maximum tolerated dose; PCNA, proliferating cell nuclear antigen; TIMP, tissue inhibitors of MMP; VEGF, vascular endothelial growth factor; vWF, von-Willebrand factor

\section{Abstract}

Although continuous low-dose (metronomic [MET]) therapy exerts anti-cancer efficacy in various cancer models, the effect of long-term MET therapy for hepatocellular carcinoma (HCC) remains unknown. This study assessed the long-term efficacy of MET on suppression of tumor growth and spontaneous metastasis in a rat model of $\mathrm{HCC}$ induced by administration of diethylnitrosamine for $16 \mathrm{wk}$. The rats were divided into 3 groups: MTD group received intraperitoneal (i.p.) injections of $40 \mathrm{mg} / \mathrm{kg}$ cyclophosphamide on days 1 , 3 , and 5 of a 21-day cycle; Control and MET groups received i.p. injections of saline and $20 \mathrm{mg} / \mathrm{kg}$ cyclophosphamide twice a week, respectively. Anti-tumor and anti-angiogenic effects and anti-metastatic mechanisms including matrix metalloproteinases (MMPs) and tissue inhibitors of MMPs (TIMPs) were evaluated. Twelve wk of MET therapy resulted in a significant reduction in intrahepatic tumors than control or MTD therapy. The MET group had fewer proliferating cell nuclear antigen-positive cells and decreased hypoxia-inducible factor-1a levels and microvessel density. Lung metastases were detected in $100 \%, 80 \%$, and $42.9 \%$ in the control, MTD, and MET groups, respectively. MET therapy significantly decreased expression of TIMP-1, MMP-2 and -9. For mediators of pro-MMP-2 activation, MET therapy induced significant suppression in the TIMP-2 and MMP-14 level. The survival in the MET group was significantly prolonged compared to the control and MTD groups. Long-term MET scheduling suppresses tumor growth and metastasis via its potent anti-angiogenic properties and a decrease in MMPs and TIMPs activities. These results provide a rationale for long-term MET dosing in future clinical trials of HCC treatment.

Keywords: cyclophosphamide; hepatocellular carcinoma; metastasis; metronomic chemotherapy

\section{Introduction}

Although there has been progress in the development of new therapies, the prognosis of hepatocellular carcinoma (HCC) still remains dismal, and only a minority of HCC patients have a chance of cure. Recently, sorafenib, an oral multiple kinase inhibitor, has been approved for the treatment of advanced HCC (Llovet et al., 2008). However, the survival benefit with sorafenib is only modest. Moreover, sorafenib is costly and often associated with adverse events during treatment in some patients. Thus, there is a crucial need for additional effective systemic therapies without major toxicities in the treatment of HCC.

$\mathrm{HCC}$ is a hypervascular tumor, and angiogenesis plays an essential role in its growth and progression. The increased cellularity due to highly proliferative tumor cells induces local hypoxia, which stimulates angiogenesis (Carmeliet and Jain, 2000; Pang and Poon, 2006). Hypoxia-inducible factor- $1 \alpha$ (HIF-1 $\alpha$ ), a master regulator of adaptive responses to hypoxia, regulates tumor cell proliferation, apoptosis, angiogenesis, and metastatic spread (Hirota, 2002; Semenza, 2003). Tumors express high levels of matrix metalloproteinases (MMPs) that degrade tissue matrix and facilitate tumor cell invasion and metastasis (Roy et al., 
2009). Earlier studies have shown that many MMPs are overexpressed in patients with $\mathrm{HCC}$, and a high level of expression of MMPs is associated with cancer progression and metastasis (Sawada et al., 2001; Ishii et al., 2003; Zhang et al., 2006). Therefore, anti-angiogenic and anti-invasive strategies could be important and prospective approaches to mitigate the growth, invasion, and metastasis of HCC.

It has been recently shown that chemotherapy with a low, non-toxic dose of drugs, but at more frequent intervals with no long rest periods, can effectively reduce tumor growth via a strong suppression of tumor angiogenesis in various tumor models (Browder et al., 2000; Gasparini, 2001; Kerbel and Kamen, 2004; Stempak et al., 2006). Moreover, this type of chemotherapeutic dosing, so-called metronomic (MET) therapy, reportedly minimizes treatment-related toxicities and significantly inhibits the development of drug resistance by destroying endothelial cells (Browder et al., 2000; Kerbel, 2007), which were the major drawbacks of conventional systemic chemotherapeutic drugs at maximum tolerated doses (MTD). In this regard, the MET strategy may become a promising approach to the treatment of HCC, because the majority of HCC patients may be potentially vulnerable to various toxicities resulting from MTD therapy because of the intrinsic liver cirrhosis.

Recently, we observed a promising chemotherapeutic efficacy against autochthonous HCC in a chemically-induced model in which rats were treated with a short course of MET therapy using cyclophosphamide (CTX) (Park et al., 2010). In this experiment, the short-term MET therapy of $6 \mathrm{wk}$ efficiently modulated tumor growth without serious toxicity, but the anti-tumor efficacy regarding an actual reduction in tumor size was modest and not significantly better than that of MTD chemotherapy. The effects and associated mechanisms of long-term MET chemotherapy on liver-confined tumor that follows the course of invasion and metastasis in the HCC model have not yet been studied.

Therefore, this study evaluated the efficacy of extended MET therapy on established hepatic tumor growth, progression, invasion, and distant metastasis in diethylnitrosamine (DEN)-treated rats. In addition, we also examined the expression of HIF- $1 \alpha$ and MMPs as associated molecular mechanisms of angiogenesis and metastasis. The present findings demonstrated amplified anti-tumor effects, prolonged survival, and anti-metastatic activities of extended MET therapy in this in vivo tumor model together with a strong anti-angiogenic effect and inhibitory activity on MMPs in tumor tissues.

\section{Results}

\section{Sequential development of cirrhosis and HCC during the administration of DEN}

During the planned DEN administration $(50 \mathrm{mg} / \mathrm{kg} /$ week), periportal fibrosis with occasional bridging fibrosis was detected on microscopic examination of the liver at $10 \mathrm{wk}$. Microscopic fibrosis became obvious from $12 \mathrm{wk}$, and distinct cirrhosis appeared at $14 \mathrm{wk}$. After $16 \mathrm{wk}$ of DEN administration, dysmorphic and dyschromic nodules with a diameter $\geq 3 \mathrm{~mm}$ in the liver were ultimately identified as HCC on histological examination (Park et al., 2010), and these nodules were used for further analysis of tumors.

\section{Effect of MET chemotherapy on hepatic tumor growth}

After 12 wk of therapy, the number and size of gross intrahepatic tumors were fewer in the MET group than the other groups (Figure 1A). The overall number of intrahepatic tumors in the MET group was significantly reduced than the control and MTD groups $(29.1 \pm 28.6$ vs. $83.2 \pm 44.9$ and $65.0 \pm 31.0 ; P=0.034$ and $P=0.048$, respectively; Figure 1B). Furthermore, on assessment of the liver and body weight, the MET group revealed a significantly decreased liver/body weight ratio than the control or MTD groups ( $4.8 \pm 0.8$ vs. $8.4 \pm 3.5$ or $6.7 \pm 0.2, P=0.05$ ), indicating a reduced tumor burden with MET therapy. The number of hepatic
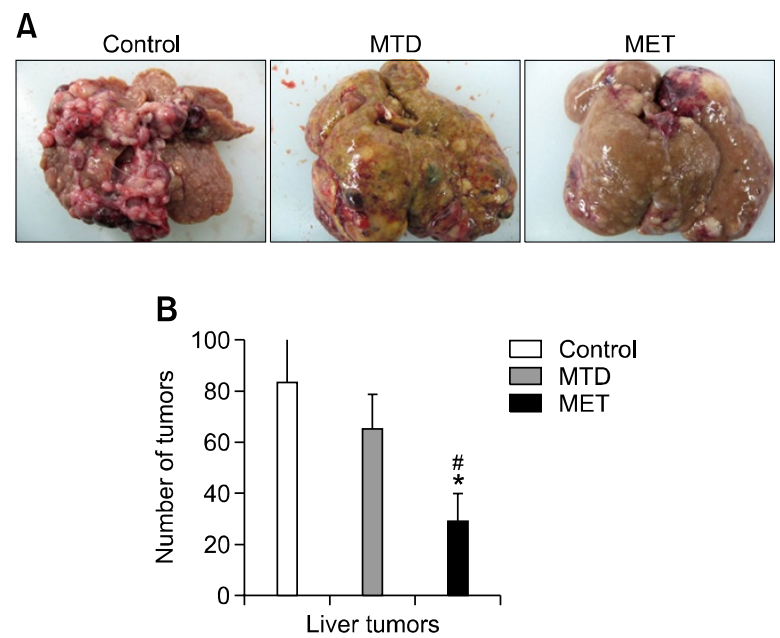

Figure 1. Liver tumors after 12 wk of treatment. (A) Macroscopic views of liver tumors of a representative rat from each group after 12 wk of treatment. (B) Number of liver tumors at 12 wk of therapy. The extent of tumors was evaluated from surviving rats at the time of sacrifice. The MET group had a significantly decreased number of liver tumors than the control and MTD groups $(29.1 \pm 28.6$ vs. $83.2 \pm 44.9$ and $65.0 \pm$ 31.0 , respectively). ${ }^{*} P<0.05$, comparison with control; ${ }^{\#} P<0.05$, comparison with MTD. 
Table 1. Liver/body weight ratio, hepatic nodular and lung metastasis appearance in surviving rats at week 12 in each group

\begin{tabular}{lccc}
\hline \multicolumn{1}{c}{ Parameters } & Control & MTD & MET \\
\hline Liver/body weight (\%) & $8.4 \pm 3.5$ & $6.7 \pm 0.2$ & $4.8 \pm 0.8$ \\
Liver tumors & $83.2 \pm 44.9$ & $65.0 \pm 31.0$ & $29.1 \pm 28.6^{\star \#}$ \\
Lung metastasis & $21.0 \pm 1.4$ & $6.0 \pm 5.2^{*}$ & $1.4 \pm 2.5^{\text {*\# }}$ \\
\hline
\end{tabular}

${ }^{*} P<0.05$, comparison with the control group. ${ }^{\sharp} P<0.05$, comparison with the MTD group.

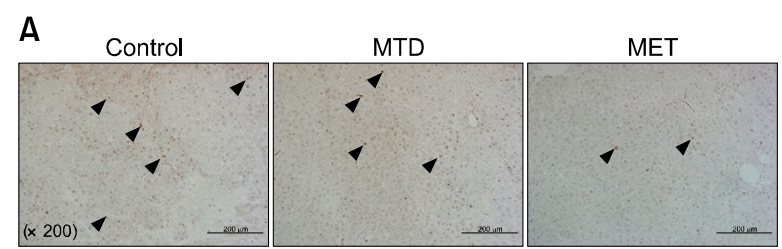

B

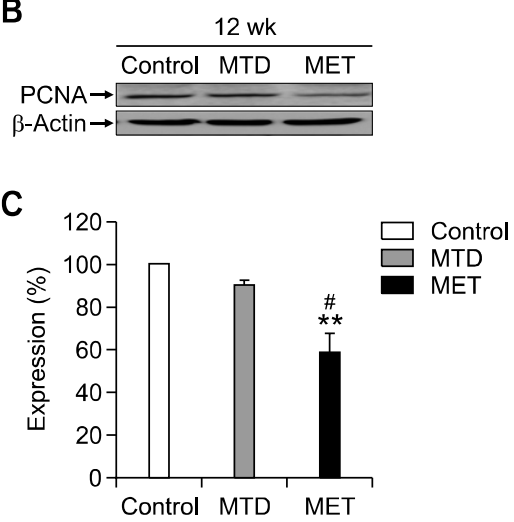

Figure 2. Effect of MET chemotherapy on proliferating tumor cells. (A) Immunohistochemistry of tumor sections prepared after 12 wk of treatment with saline (control), MTD, and MET, respectively. Tumor sections were stained with an anti-PCNA antibody to detect proliferating cells (arrowheads) (magnification $\times 200)$. (B) Immunoblot analysis of expression of PCNA in protein levels after 12 wk of treatment. $\beta$-actin was used as a loading control. (C) Bar graph of the band intensities on PCNA expression. PCNA expression was quantified by densitometry with an Image software Gauge 4.0 program. ${ }^{* *} P<0.01$, comparison with control; ${ }^{~} P<0.05$, comparison with MTD.

nodules and liver/body ratio in each group are summarized in Table 1.

\section{Suppression of tumor cell proliferation by MET chemotherapy}

The MET therapy provided fewer PCNA (proliferating cell nuclear antigen)-positive cells compared to the control or MTD therapy groups (Figure 2A). We then showed that the MET chemotherapy group had down-regulated expression of PCNA compared with the other groups, as confirmed by an analysis of band densities (Figure 2B). Quantitation of PCNA-positive cells showed a significantly decreased number of proliferating cells by MET therapy compared with the control and MTD the-
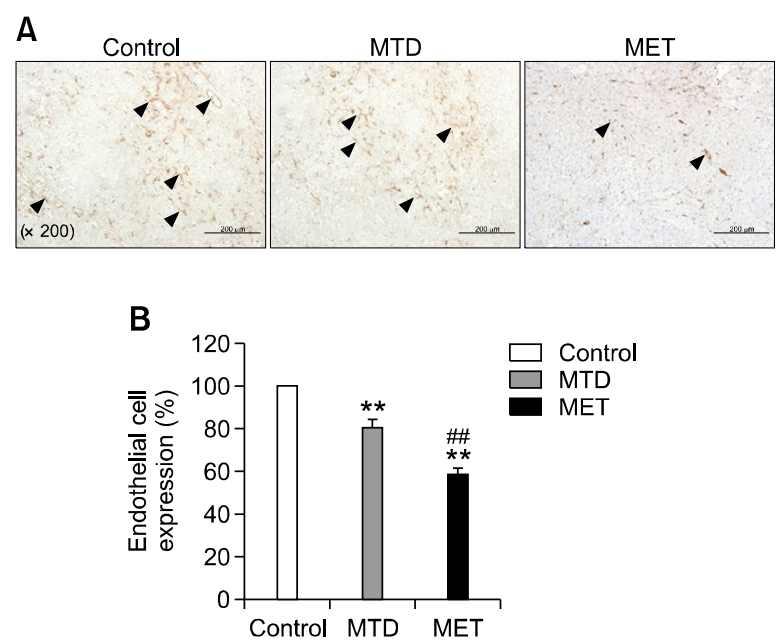

Figure 3. Effect of MET chemotherapy on tumor angiogenesis. (A) Expression of VWF-positive endothelial cells (arrowheads) in tumor sections after treatment with saline (control), MTD and MET at 12 wk, respectively (magnification $\times 200$ ). (B) Bar graph of the mean percentage of vWF-positive cells in each group. Five fields per slide, and at least three slides per group, were examined. ${ }^{* *} P<0.01$, comparison with control; ${ }^{\#} P<0.01$, comparison with MTD.

rapy $(P=0.004$ and $P=0.015$, respectively; Figure 2C).

\section{Anti-angiogenic effect of MET chemotherapy}

As noted in Figure 3A, tumor microvessel density (MVD) was markedly decreased by MET chemotherapy. Although both treatments decreased angiogenesis compared with the control group, the MET therapy provided a greater decrease in endothelial cell expression than the MTD chemotherapy $(P=0.002$; Figure $3 \mathrm{~B})$. Using quantitative RT-PCR, we next examined the effect of treatment on HIF- $1 \alpha$, a regulator of tumor angiogenesis in tumors, and showed that the expression of HIF-1 $\alpha$ was significantly decreased by extended treatment of MET dosing up to $12 \mathrm{wk}$ compared to the other therapies $(P=0.021$; Figures $6 \mathrm{~A}-\mathrm{C})$.

\section{Suppression of lung metastasis by MET chemotherapy}

Since control rats often died due to tumor progre- 


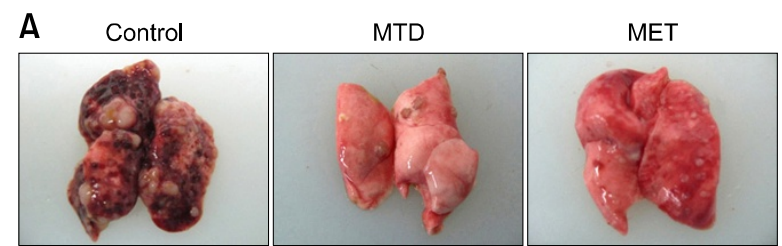

B

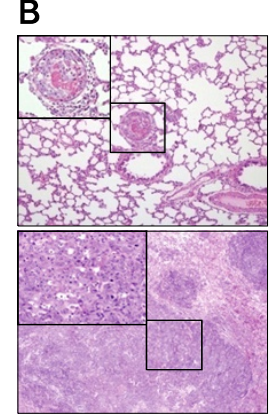

c

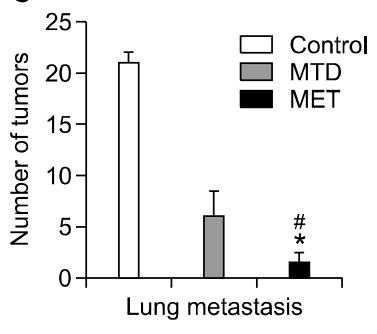

Figure 4. Spontaneous lung metastases from hepatic tumors. Surviving rats in each group were sacrificed at 12 wk of treatment, and macroscopic pulmonary nodules were counted. (A) Gross findings of lungs of a representative rat from each group after treatment for 12 wk. (B) Tumor cell invasion in a vessel of the lung (H\&E staining, magnification $\times 100$ and 400) and hepatocellular carcinoma-like cells shown in the lung (H\&E staining, magnification $\times 200$ and 400). (C) Number of lung metastasis at 12 wk of therapy. Spontaneous lung metastases from HCC after 12 wk were significantly fewer in the MET group $(1.4 \pm 2.5)$ than the control $(21.0 \pm 1.4)$ and $\operatorname{MTD}(6.0 \pm 5.2)$ groups. ${ }^{*} P<0.05$, comparison with control; ${ }^{\#} P<0.05$, comparison with MTD.

ssion during the follow-up, only two rats surviving up to 12 wk could be evaluated for analysis in the control group. At $12 \mathrm{wk}$, all rats $(100 \%, 2 / 2)$ in the control and $80 \%(4 / 5)$ in the MTD groups had macroscopic lung metastases, while only $42.9 \%$ (3/7) of the rats in the MET group had lung metastases (Figure 4). Spontaneous lung metastases from HCC after 12 wk were significantly fewer in the MET group than the control $(1.4 \pm 2.5$ vs. 21.0 $\pm 1.4, P=0.025)$ and MTD $(1.4 \pm 2.5$ vs. $6.0 \pm$ $5.2, P=0.043$ ) groups (Figure $4 \mathrm{C}$; Table 1 ).

\section{Activities of MMPs and TIMPs}

To obtain more detailed information regarding the anti-metastatic mechanism of therapy, the activities of MMPs and TIMPs were examined at different time points of 6 and 12 wk. Five rats in each group were sacrificed, and tumors of similar size (approximately $7 \mathrm{~mm}$ in diameter) were used for comparison. As shown by gelatin zymography (Figure $5 A$ ), MET therapy strongly reduced the activities of gelatinases (MMP-2 and -9), which was greater suppression than MTD therapy, while the active form of MMP-9 was largely undetectable in all groups. After $6 \mathrm{wk}$, both therapies decreased the activities of MMPs, but MET therapy yielded a
A

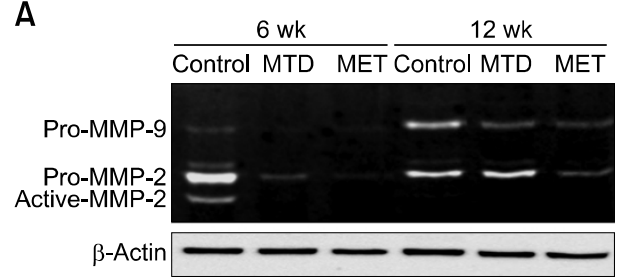

B
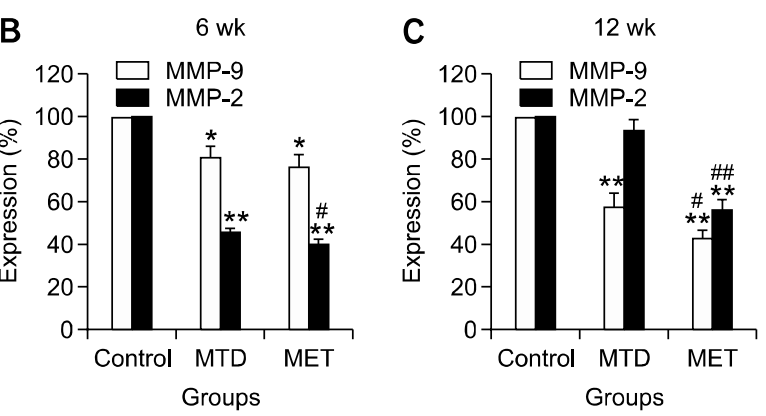

Figure 5. Gelatin zymographic analysis of MMP-2 and -9 activities. (A) Gelatin zymography of pro-MMP-9, pro-MMP-2 and active MMP-2 after treatment for 6 and $12 \mathrm{wk}$. The protein concentration was determined by the Bradford protein assay, and equal amounts of protein were applied to each lane $(30 \mu \mathrm{g} / \mathrm{lane})$. The picture shows a representative gel of three comparable experiments. MMP activities are visualized as white bands, corresponding to proform MMP-9 (92 KDa), proform MMP-2 (72 KDa) and active MMP-2 (62 KDa). $\beta$-actin was used as a loading control. ( $\mathrm{B}$ and C) Bar graphs of the band intensities on MMP-9 and MMP-2 in protein levels at 6 and 12 wk of therapy. Expression of MMPs was quantified by densitometry with an Image software Gauge 4.0 program. ${ }^{*} P<0.05$, ${ }^{* \star} P<0.01$, comparison with control; ${ }^{\#} P<0.05,{ }^{\#} P<0.01$, comparison with MTD.

greater decrease in the MMP level than MTD therapy (Figure 5A). The intensity of gelatinolytic bands for both MMP-2 and MMP-9 was significantly lower in the MET group than the MTD group throughout the duration of treatment (all $P<0.05$ ), except the MMP-9 level at 6 wk (Figure 5B). The activity of MMP-2 in the MTD group significantly increased thereafter during extension of treatment up to $12 \mathrm{wk}$. The expression level of MMP transcripts in tumor also correlated well with the zymographic findings. As shown in Figure 6, the expression of MMP transcripts was more reduced by MET therapy. Particularly, the level of MMP-2 transcripts was significantly lower after extended MET therapy compared to MTD therapy $(P=0.05$; Figure $6 \mathrm{C})$. Because the process of MMP-2 activation is mediated by the formation of a complex between membrane type 1 (MT1)-MMP (MMP-14), TIMP-2, and pro-MMP-2 (Butler et al., 1998; Kinoshita et al., 1998; Seiki, 1999), we thus attempted to examine the expression levels of MMP-14 and TIMP-2 within the tumor areas using quantitative RT-PCR. As a result, the expression of MMP-14 and TIMP-2 was significantly decreased by MET therapy when compared with the control (all $P<$ 

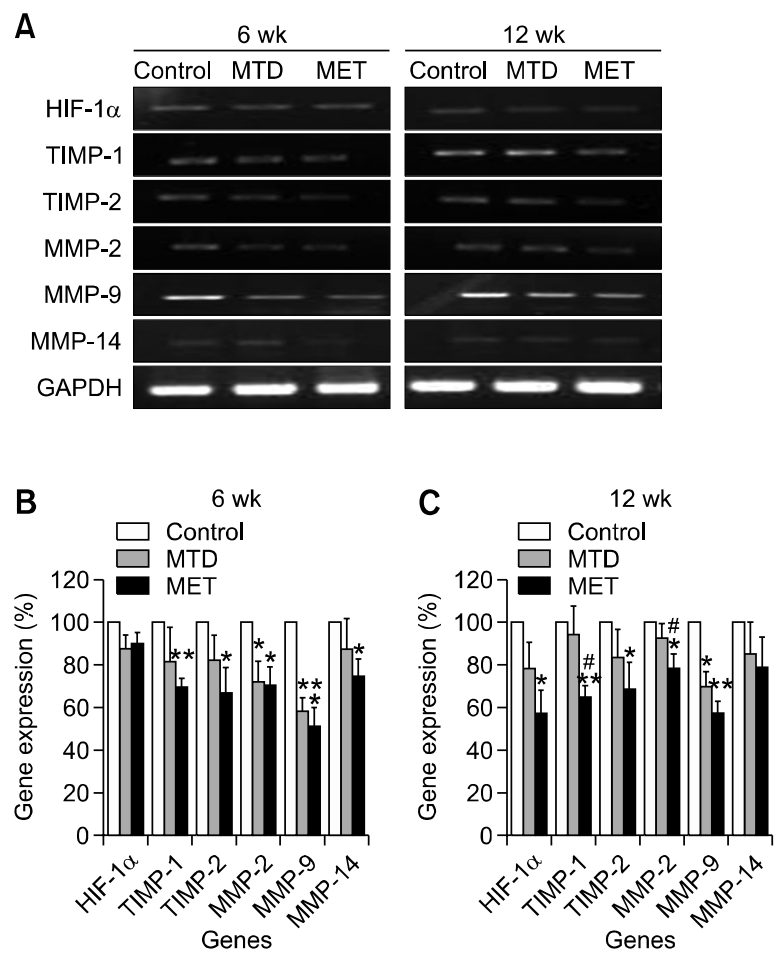

Figure 6. Expression of HIF-1 $\alpha$, MMPs, and TIMPs in mRNA levels after treatment for 6 and 12 wk. (A) RT-PCR was performed to examine these factors. The expression of each lane was normalized to the level of GAPDH. The experiment was done in triplicate. (B and $C)$ Bar graphs of the band intensities on HIF-1 $\alpha$, MMPs, and TIMPs in mRNA levels. Expression of these factors was quantified by densitometry with an Image software Gauge 4.0 program. ${ }^{*} P<0.05,{ }^{* *} P<0.01$, comparison with control; ${ }^{\sharp} P<0.05$, comparison with MTD.

0.05), except for the level of MMP-14 at $12 \mathrm{wk}$. In contrast, the suppressive effect of MMP-14 or TIMP-2 was not observed during MTD therapy (Figures 6B-C). In addition, we measured the level of TIMP-1 that was suggested to be another metastatic marker in HCC (Nakatsukasa et al., 1996), and found a dramatic reduction in the expression level of TIMP-1 by MET therapy. The level of TIMP-1 after 12 wh of MET therapy was significantly suppressed more than the control and MTD therapy groups $(P=0.008$ and $P=0.045$, respectively; Figure 6C).

\section{Prolonged survival with MET chemotherapy}

The median survival time of the MET group was 68 days, which was significantly better than the control (39 days) or MTD group (52 days) (log-rank test: $P$ $=0.001$ and $P=0.014$, respectively). In contrast, the MTD group had no significant survival benefit when compared with the control group $(P=0.356$; Figure 7).

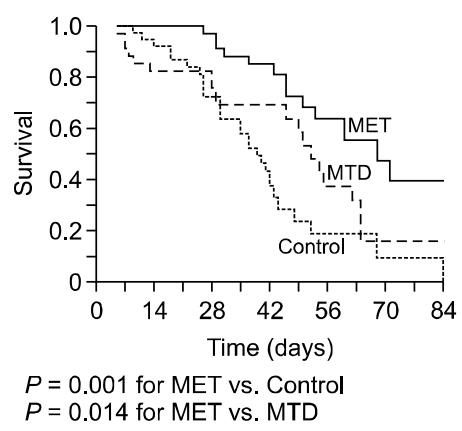

Figure 7. Overall survival in the three groups. The overall survival time was significantly prolonged in the MET group than in the control $(P=$ $0.001)$ and MTD ( $P=0.014$; log-rank test) groups.

\section{Discussion}

The present study investigated the enhanced antitumor effect of long-term MET therapy, primarily focusing on the inhibitory effect of spontaneous metastasis from well-established HCC in an autochthonous model. The results demonstrated that extended MET chemotherapy showed excellent efficacy in suppressing intrahepatic tumor growth and metastasis of HCC. This is noteworthy because despite the promising anti-angiogenic properties noted in our previous work, a short-term MET treatment $(6 \mathrm{wk})$ failed to show a significant intrahepatic tumor reduction as compared with MTD therapy (Park et al., 2010). Consistently, the inhibitory effect of MET on tumor cell proliferation was more significant with extension of treatment up to $12 \mathrm{wk}$, while it was not different between the MET and MTD groups at 6 wk of therapy (Park et al., 2010). These findings indicate that with the extension of treatment, the tumor suppressive effect of MET scheduling is further amplified via more enhanced anti-proliferative and anti-angiogenic properties, which were significantly better than achieved with MTD therapy. Thus, this supports the previous findings that an actual effect of MET therapy is achievable when exposed continuously for longer periods of time, but not for shorter periods of time (Bocci et al., 2002).

The principal mechanism of MET dosing to treat cancers is its potent anti-angiogenic activity by targeting both dividing endothelial cells in the tumor neovasculature and bone marrow-derived circulating endothelial progenitor cells (Kerbel, 2007). As expected, extended MET therapy resulted in a marked decrease in MVD on immunohistochemical analysis, which was more significant than MTD treatment and persisted up to $12 \mathrm{wk}$. Moreover, long-term MET therapy exerted a significant inhibition of expression of HIF-1 $\alpha$, known to be a key modulator of angiogenesis. These findings 
further confirm the strong anti-angiogenic property of MET therapy as the main tumor-suppressive mechanism.

One of the key findings in this study is that extended MET therapy significantly suppressed spontaneous pulmonary metastasis from HCC. Among the MMPs as a major factor for metastasis, the gelatinases MMP-2 and -9 are the most studied MMPs and clearly associated with the processes of cancer invasion and metastasis (Egeblad and Werb, 2002). The inhibition of either MMP-2 or -9 activities reportedly resulted in the reduction in tumor metastasis in in vivo models (Itoh et al., $1998,1999)$. In this analysis, we observed that extended MET therapy markedly suppresses the expression of both MMP-2 and -9 activities. Thus, these results suggest that the possible mechanism of suppression of pulmonary metastasis by MET may involve the inhibition of these MMPs activities.

The MMPs are synthesized as inactive zymogens and activated by proteinase cleavage. Specially, Pro-MMP-2 is known to be activated at the cell surface through a unique multistep pathway that involves MMP-14 (MT1-MMP) and the tissue inhibitor of TIMP-2 (Egeblad and Werb, 2002). In this experiment, extended MET therapy also induced a significant reduction in the expression of both MMP-14 and TIMP-2, as evidenced by quantitative RT-PCR. In addition, MET treatment significantly decreased the expression of TIMP-1. Although its role is not completely understood in HCC, TIMP-1 is reportedly overexpressed in HCC tissues, and suggested to be a potential marker of metastasis to the lung (Nakatsukasa et al., 1996; Matsumoto et al., 2004). Thus, the overall findings imply that the anti-metastatic effects of MET therapy may not simply be due to the suppression of primary tumor growth, but rather an additive effect of direct antimetastatic potentials that are, at least in part, mediated through the down-regulation of MMP and TIMP activities in a coordinate manner by MET therapy.

The major advantages of metronomic strategy in cancer treatment include its lower toxicities, as also seen in this HCC model (Park et al., 2010), and the possibility of combining it with other anticancer therapies (Kerbel, 2007). These properties of MET dosing remain the major therapeutic merits in treating HCC patients with significant co-morbid conditions, such as underlying cirrhosis. In particular, angiogenesis is an essential process to the development of $\mathrm{HCC}$, and there are currently several molecular targeted agents with encouraging therapeutic efficacy in tumor control. In this clinical context, metronomic dosing of chemotherapeutic agents is expected to be a promising option in the future management of HCC. However, the optimal biologic dosing and scheduling still remain to be determined in the presence of underlying liver cirrhosis, because the metabolism and activation of chemotherapeutic drugs can be potentially affected by liver function (Superfin et al., 2007).

In conclusion, extended MET therapy resulted in a significant suppression of intrahepatic tumor growth and metastasis, and prolonged survival via strong anti-proliferative, anti-angiogenic and antimetastatic properties in an autochthonous model of HCC. Our results support the potential for longterm use of metronomic scheduling, which can be achieved in practice without treatment interruptions. Given the modest efficacy and frequent side effects of sorafenib, the only drug currently approved as a systemic agent for HCC, metronomic chemotherapeutic scheduling is a promising approach without major toxicities in the treatment of HCC. Clinical trials using this approach are needed in the near future.

\section{Methods}

\section{Experimental design}

Male Sprague Dawley (SD) rats were initiated with intraperitoneal (i.p.) injections of DEN (Sigma Chemical Co., St. Louis, MO) at $50 \mathrm{mg} / \mathrm{kg}$ body weight in $0.9 \% \mathrm{NaCl}$ once a week for 16 wk for induction of HCC (Schiffer et al., 2005; Park et al., 2010). After 8 wk, two rats were killed at intervals of 2 wk until the development of HCC. Therapy began at $17 \mathrm{wk}$ and the rats were sacrificed for experiments 6 and 12 wk after therapy in each group. The rats were randomly divided into three groups: the MTD group received a $40 \mathrm{mg} / \mathrm{kg}$ CTX (Sigma Chemical Co.) i.p. injection on days 1,3 , and 5 of a 21-day cycle; the control group received an i.p. injection of saline twice a week; and the MET group received a $20 \mathrm{mg} / \mathrm{kg}$ CTX i.p. injection twice a week. Animal care and the experiments followed the guidelines for the Care and Use of Laboratory Animals from the Research Supporting Center for Medical Science of The Catholic University of Korea.

\section{Macroscopic and microscopic observations}

To examine tumor growth, after the rats were killed, tissues of whole liver and lung were removed, fixed, and were sliced at $2 \mathrm{~mm}$-thicknesses. Dyschromatic and dysmorphic nodules of $\geq 3 \mathrm{~mm}$ in the liver and lung were counted by 2 independent investigators. The tissue samples were fixed in $10 \%$ formalin for $24 \mathrm{~h}$ before being embedded in paraffin. The paraffin-embedded sections were cut into 3 $\mu \mathrm{m}$ thicknesses, dewaxed, dehydrated, and stained with hematoxylin and eosin (H\&E). One pathologist (C.K.J.) blinded of experimental detail examined the sections. 


\section{Total RNA extraction and semi-quantitative RT-PCR}

Total RNA was extracted from frozen tissues using TRIZOL reagent following the manufacturer's instructions. RNAs were quantified by absorbance at $260 \mathrm{~nm}$ and then reverse-transcribed to single-strand cDNAs using random primer (TaKaRa Bio, Shiga, Japan) with a SuperScript ${ }^{\mathrm{TM}}$ ॥ First Strand Synthesis System (Invitrogen). $2 \mu \mathrm{g}$ of total RNA was amplified by polymerase chain reaction (PCR) and the cDNAs were amplified. The sequences of all the primers and conditions are listed in Supplementary Table 1.

\section{Immunohistochemical analysis}

For immunohistochemical staining using an antibody to PCNA and von-Willebrand factor (VWF), the paraffin sections were dewaxed in xylene and rehydrated through a graded alcohol series. To block endogenous peroxidase activity, the sections were quenched in $3 \%$ hydrogen peroxide for $10 \mathrm{~min}$, and antigen retrieval was carried out in citrate buffer ( $\mathrm{pH} \mathrm{6.0)}$ by a 30 min microwave treatment. Next, slides were blocked with normal serum blocking solution (DakoCytomation, Glostrup, Denmark) at room temperature for 30 minutes, followed by incubation with primary antibody (PCNA, vWF) at $4^{\circ} \mathrm{C}$ for overnight. After rinsing the slides, the secondary antibody conjugated in a biotinylated link universal solution (Dakocytomation, Denmark) was applied at room temperature for $2 \mathrm{~h}$. Then, the streptavidin-peroxidase solution (Dakocytomation, Denmark) was applied and the slides were incubated for $1 \mathrm{~h}$ at room temperature. Horseradish peroxidase was detected with 3 , 3-diaminobenzine (Vector Laboratories, Burlingame, CA) and counterstained with hematoxylin. Stained sections were counted in 5 high density fields at 400 magnifications, and the mean endothelial cells density was recorded.

\section{Immunoblot analysis}

The tissues were homogenized in t-per tissue protein extraction buffer (Pierce, Rockford, IL). The lysates were cleared by centrifugation at $10,000 \times \mathrm{g}$ for $30 \mathrm{~min}$ at $4^{\circ} \mathrm{C}$, and the supernatant kept frozen at $-70^{\circ} \mathrm{C}$ until use. The protein concentration was determined by Bradford assay (Bio-Rad Laboratories, Hercules, CA) with bovine serum albumin as the standard. Proteins $(40 \mu \mathrm{g})$ were separated by $8 \%$ SDS-polyacrylamide gel electrophoresis (PAGE) and transferred to nitrocellulose membranes (Whatman, Maidstone, Kent, UK) for $1 \mathrm{hr}$ at $4^{\circ} \mathrm{C}$. Then, membranes were blocked with $5 \%$ skim milk in PBS at room temperature for $30 \mathrm{~min}$. The membranes were incubated with monoclonal anti- $\beta$-actin (Sigma-Aldrich) and PCNA (Abcam, UK) at $4^{\circ} \mathrm{C}$ overnight. Each membrane was washed 3 times with $0.05 \%$ Tween-20 contained TBS (TBS-T), following incubated with horseradish peroxidase-conjugated anti-mouse secondary antibody (Amersham Bosciences, Buckinghamshire, UK). Then, the specific protein bands were visualized with Enhanced Chemiluminescent system (Amersham, Biosciences), according to the manufacturer's instructions. The relative intensity of the bands was analyzed using the Luminescent Image Analyzer LAS-4000 Plus and Image software Gauge 4.0 (FUJI Photo Film, Co. Ltd, Minamiashigara, Japan).

\section{Zymography}

Expression of the proform and activated form of MMP-2 and -9 was analyzed by zymography. The samples taken from tumors of similar sizes in each group were then loaded onto $8 \%$ SDS PAGE electrophoresis gels containing $0.1 \%$ gelatin. After protein separation by electrophoresis under non-reducing conditions, the gel was washed with $2.5 \%$ Triton X-100 3 times for 30 minutes and then incubated for $24 \mathrm{~h}$ in substrate activation buffer (50 $\mathrm{mM}$ Tris- $\mathrm{HCl}\left[\mathrm{pH}\right.$ 7.6], $150 \mathrm{mM} \mathrm{NaCl}, 10 \mathrm{mM} \mathrm{CaCl}_{2}$, and $0.002 \% \mathrm{ZnCl} 2)$ at $37^{\circ} \mathrm{C}$. The gels were stained with Coomassie blue $(0.5 \%$ Coomassie R-250, $45 \%$ methanol, and $10 \%$ acetic acid) for $2 \mathrm{~h}$ and destained in washing solution (45\% methanol, and $10 \%$ acetic acid) as necessary. White bands on a blue background indicated zones of digestion corresponding to the presence of different pro-MMPs and activated MMPs on the basis of their molecular weight. The relative intensity of signals was analyzed and compared.

\section{Statistics}

Continuous variables were compared with the $t$-test or ANOVA if normally distributed, and the Mann-Whitney test or Kruskal-Wallis test if non-parametric. Survival curves were plotted using the Kaplan-Meier method, and the differences were analyzed by the log-rank statistic. $P$ values $<0.05$ were considered to be statistically significant. All statistical analyses were performed using SPSS software (version 15.0, Chicago, IL).

\section{Acknowledgements}

This research was supported by The Korean Association for the Study of the Liver, Grant No.0620390 from the National R \& D Program for Cancer Control, Ministry for Health, Welfare and Family Affairs, Republic of Korea.

\section{Supplemental data}

Supplemental data include a table and can be found with this article online at http://e-emm.or.kr/article/article_files/ SP-43-5-06.pdf.

\section{References}

Bocci G, Nicolaou KC, Kerbel RS. Protracted low-dose effects on human endothelial cell proliferation and survival in vitro reveal a selective antiangiogenic window for various chemotherapeutic drugs. Cancer Res 2002;62:6938-43

Browder T, Butterfield CE, Kraling BM, Shi B, Marshall B, O'Reilly MS, Folkman J. Antiangiogenic scheduling of chemotherapy improves efficacy against experimental drug-resistant cancer. Cancer Res 2000;60:1878-86

Butler GS, Butler MJ, Atkinson SJ, Will H, Tamura T, Schade van Westrum S, Crabbe T, Clements J, d'Ortho MP, Murphy G. The TIMP2 membrane type 1 metalloproteinase "receptor" regulates the concentration and efficient activation of progelatinase A. A kinetic study. J Biol Chem 1998;273: 


\section{$871-80$}

Carmeliet P, Jain RK. Angiogenesis in cancer and other diseases. Nature 2000;407:249-57

Egeblad M, Werb Z. New functions for the matrix metalloproteinases in cancer progression. Nat Rev Cancer 2002;2:161-74

Gasparini G. Metronomic scheduling: the future of chemotherapy? Lancet Oncol 2001;2:733-40

Hirota K. Hypoxia-inducible factor 1, a master transcription factor of cellular hypoxic gene expression. J Anesth 2002;16:150-9

Ishii Y, Nakasato Y, Kobayashi S, Yamazaki Y, Aoki T. A study on angiogenesis-related matrix metalloproteinase networks in primary hepatocellular carcinoma. J Exp Clin Cancer Res 2003;22:461-70

Itoh T, Tanioka M, Yoshida H, Yoshioka T, Nishimoto H, Itohara S. Reduced angiogenesis and tumor progression in gelatinase A-deficient mice. Cancer Res 1998;58:1048-51

Itoh T, Tanioka M, Matsuda $\mathrm{H}$, Nishimoto $\mathrm{H}$, Yoshioka $\mathrm{T}$, Suzuki R, Uehira M. Experimental metastasis is suppressed in MMP-9-deficient mice. Clin Exp Metastasis 1999;17: 177-81

Kerbel RS. Improving conventional or low dose metronomic chemotherapy with targeted antiangiogenic drugs. Cancer Res Treat 2007;39:150-9

Kerbel RS, Kamen BA. The anti-angiogenic basis of metronomic chemotherapy. Nat Rev Cancer 2004;4:423-36

Kinoshita T, Sato H, Okada A, Ohuchi E, Imai K, Okada Y, Seiki M. TIMP-2 promotes activation of progelatinase $A$ by membrane-type 1 matrix metalloproteinase immobilized on agarose beads. J Biol Chem 1998;273:16098-103

Llovet JM, Ricci S, Mazzaferro V, Hilgard P, Gane E, Blanc JF, de Oliveira AC, Santoro A, Raoul JL, Forner A, Schwartz M, Porta C, Zeuzem S, Bolondi L, Greten TF, Galle PR, Seitz JF, Borbath I, Haussinger D, Giannaris T, Shan M, Moscovici M, Voliotis D, Bruix J. Sorafenib in advanced hepatocellular carcinoma. N Engl J Med 2008;359:378-90

Matsumoto E, Nakatsukasa H, Nouso K, Kobayashi $\mathrm{Y}$, Nakamura S, Suzuki M, Takuma Y, Tanaka H, Fujikawa T, Shiratori Y. Increased levels of tissue inhibitor of metalloproteinase-1 in human hepatocellular carcinoma. Liver Int 2004;24:379-83
Nakatsukasa H, Ashida K, Higashi T, Ohguchi S, Tsuboi S, Hino N, Nouso K, Urabe Y, Kinugasa N, Yoshida K, Uematsu S, Ishizaki M, Kobayashi Y, Tsuji T. Cellular distribution of transcripts for tissue inhibitor of metalloproteinases 1 and 2 in human hepatocellular carcinomas. Hepatology 1996;24: 82-8

Pang R, Poon RT. Angiogenesis and antiangiogenic therapy in hepatocellular carcinoma. Cancer Lett 2006;242:151-67

Park ST, Jang JW, Kim GD, Park JA, Hur W, Woo HY, Kim JD, Kwon JH, Yoo CR, Bae SH, Choi JY, Yoon SK. Beneficial effect of metronomic chemotherapy on tumor suppression and survival in a rat model of hepatocellular carcinoma with liver cirrhosis. Cancer Chemother Pharmacol 2010;65: 1029-37

Roy R, Yang J, Moses MA. Matrix metalloproteinases as novel biomarkers and potential therapeutic targets in human cancer. J Clin Oncol 2009;27:5287-97

Sawada S, Murakami K, Murata J, Tsukada K, Saiki I. Accumulation of extracellular matrix in the liver induces high metastatic potential of hepatocellular carcinoma to the lung. Int J Oncol 2001;19:65-70

Schiffer E, Housset C, Cacheux W, Wendum D, DesboisMouthon C, Rey C, Clergue F, Poupon R, Barbu V, Rosmorduc O. Gefitinib, an EGFR inhibitor, prevents hepatocellular carcinoma development in the rat liver with cirrhosis. Hepatology 2005;41:307-14

Seiki M. Membrane-type matrix metalloproteinases. APMIS 1999;107:137-43

Semenza GL. Targeting HIF-1 for cancer therapy. Nat Rev Cancer 2003;3:721-32

Stempak D, Seely D, Baruchel S. Metronomic dosing of chemotherapy: applications in pediatric oncology. Cancer Invest 2006;24:432-43

Superfin D, lannucci AA, Davies AM. Commentary: Oncologic drugs in patients with organ dysfunction: a summary. Oncologist 2007;12:1070-83

Zhang Q, Chen X, Zhou J, Zhang L, Zhao Q, Chen G, Xu J, Qian F, Chen Z. CD147, MMP-2, MMP-9 and MVD-CD34 are significant predictors of recurrence after liver transplantation in hepatocellular carcinoma patients. Cancer Biol Ther 2006;5:808-14 